\title{
Clinical pathological analysis of newly diagnosed malignant pelvic tumors during pregnancy
}

\author{
Ying Jiang", Jing Zhao", Ling Ding, Jinfeng Li, Shuzhen Wang \\ Department of Obstetrics and Gynecology, Beijing Chao-Yang Hospital, Capital Medical University, Beijing, China \\ Contributions: (I) Conception and design: S Wang, Y Jiang; (II) Administrative support: S Wang; (III) Provision of study materials or patients: Y Jiang, \\ J Zhao, J Li; (IV) Collection and assembly of data: L Ding; (V) Data analysis and interpretation: Y Jiang, S Wang, Y Jiang; (VI) Manuscript writing: \\ All authors; (VII) Final approval of manuscript: All authors. \\ \#These authors contributed equally to this work. \\ Correspondence to: Shuzhen Wang. Department of Obstetrics and Gynecology, Beijing Chao-Yang Hospital, Capital Medical University, No.8 \\ Gongren TiyuchangNanlu, Chaoyang District, Beijing, China. Email: darrywang2003@163.com.
}

Background: This study sought to investigate the pathological and clinical characteristics of newly developed malignant pelvic tumors during pregnancy.

Methods: A retrospective analysis was conducted of 20 patients with newly developed malignant pelvic tumors during pregnancy who were admitted to hospital from January 1, 2012 to March 31, 2020. The patients were grouped according to the histological tumor source, evaluated using a chi-square test and Kaplan-Meier analysis, and their clinical and pathological characteristics were examined.

Results: Of the 20 patients, 14 had primary tumors and 6 had metastatic tumors. The average age of the patients in the metastatic tumor group was higher than that of patients in the primary tumor group $(\mathrm{P}=0.038)$. Notably, patients in both groups were at an advanced tumor stage when they were diagnosed. A significant difference between the 2 groups $(\mathrm{P}<0.001)$ was found in the clinical presents and the outcomes. The survival rates of the 2 groups differed significantly $(\mathrm{P}<0.001)$. Patients in the primary tumor group all survived without tumors. Conversely, only 1 patient in the metastatic tumor group survived without a tumor during the follow-up period, and 5 patients in this group did not survive. The metastatic rates of the examined placentas were $1 / 6$ and $3 / 5$ for the primary and metastatic tumor groups, respectively $(\mathrm{P}=0.545)$. The histological distribution of the newly developed malignant pelvic tumors during pregnancy was diverse, and different to those in the non-pregnancy period. Additionally, 6 patients had metastatic tumors, and the proportion of metastatic tumors was significantly higher than that of non-pregnancy patients $(\mathrm{P}<0.001$; $3.53 \%$ vs. $30 \%)$. Of the 8 patients with primary ovarian tumors, 5 had borderline tumors (62.5\%), and the proportion of borderline tumors was significantly higher than that of non-pregnant patients $(9.61 \% v s .5 / 8)$ in the same period.

Conclusions: Newly diagnosed malignant pelvic tumors during pregnancy were different from the nonpregnant in the tissue origin, pathological grade, clinical presenting, and outcomes. The metastatic tumors pose a serious threat to the lives and health of pregnant women

Keywords: Pregnancy; malignant pelvic tumors; metastatic tumors; prognosis

Submitted Apr 02, 2021. Accepted for publication Jun 04, 2021.

doi: $10.21037 /$ tcr-21-717

View this article at: https://dx.doi.org/10.21037/tcr-21-717

\section{Introduction}

With the maternal ageing, a better national health-care system for pregnant women, more newly development pelvic malignant tumors were diagnosed (1). According to statistical data from the United States, the incidence of malignant tumors during pregnancy represents 
approximately $1 \%$ (2) of total pregnancies. Based on the British National Survey, the incidence of cancer in women during pregnancy or within 1 year of delivery is $48 \%$ higher than that of non-pregnant women of the same age (3). Due to limited clinical data and few systematic studies, no accurate data currently exists on its incidence in China.

Previous studies have shown that the common pathological types of malignant tumors in pregnancy include breast cancer, cervical cancer, and Hodgkin's lymphoma (4). The incidence of gastrointestinal tumors during pregnancy is low; however, patients with gastric and intestinal cancers during pregnancy account for $0.025-0.1 \%$ (5) and $1 / 13,000$ (6) of all pregnant women may suffer from gastrointestinal cancer in pregnancy. Very little research appears to have been conducted on pancreatic or liver cancer development during pregnancy. However, a clinical study showed that most pelvic metastatic malignant cancers during pregnancy originate from the gastrointestinal tract and hematological system. During pregnancy, borderline tumors are difficult to identify, malignant tumors are easily misdiagnosed and missed, and some tumors may even deteriorate faster, as the physiological changes that occur during pregnancy often conceal the symptoms of tumors (7). Additionally, uterus enlargement and physiological changes of the pelvic organs during pregnancy interfere with the accuracy and sensitivity of imaging diagnosis (8). Thus, clinicians need to be very careful in the diagnosis of pelvic malignancies in pregnancy, carefully identify tumors, and provide early diagnoses.

Due to the low incidence of newly developed pelvic malignant tumors during pregnancy, and the unavailability of clinical studies with large sample sizes, doctors mainly refer to clinical studies with small sample sizes and expert consensus to determine current treatment (9). A clear pathological classification is essential if a reasonable diagnosis and treatment strategy is to be developed. Recent studies have focused on the diagnosis and treatment of ovarian and cervical cancer during pregnancy $(10,11)$; however, there is a lack of systematic research on newly developed malignant pelvic tumors during pregnancy. This study undertook a retrospective case analysis to investigate the clinicopathological data of 20 patients with newly developed malignant pelvic tumors during pregnancy at the hospital of this study. Additionally, this study sought to systematically analyze the pathological and clinical characteristics of malignant pelvic tumors and provide references for the early diagnosis and reasonable treatment of the disease.

We present the following article in accordance with the STROBE reporting checklist (available at https://dx.doi. org/10.21037/tcr-21-717).

\section{Methods}

\section{Study subjects}

The data of 20 patients with newly diagnosed malignant pelvic tumors during pregnancy who had been admitted to Beijing Chao-Yang Hospital, Capital Medical University, Beijing, China from January 1, 2012 to March 31, 2020 were retrieved from the electronic medical record database of the hospital. 737 patients with non-gestational malignant pelvic tumors were also treated at the hospital during the same period. Of these 737 patients, 26 (3.53\%) had metastatic tumors. and of 489 patients of primary ovarian malignant tumors, $47(9.61 \%)$ classified as borderline cancers.

To be eligible to participate in this study, patients had to meet the following inclusion criteria: (I) have been diagnosed with newly developed pelvic malignant and borderline tumors by the patient's symptoms, pre-pregnancy medical history, physical examination results, family medical history, physical examination findings, tumor markers and morphological characteristics of the pregnant mass, which was assessed by pelvic examination, ultrasonography, pelvic MRI and surgical pathological diagnosis during pregnancy and within 1 year after delivery; and (II) have undergone tumor treatment at Beijing Chao-Yang Hospital, Capital Medical University and have complete medical records. According to different pathological diagnoses, enrolled patients were divided into a primary tumor group (comprising 14 patients) and a metastatic tumor group (comprising 6 patients). Patients were excluded from the study if they met any of the following exclusion criteria: (I) had tumors that were not newly developed during pregnancy; (II) had undergone or ended the treatment of malignant pelvic tumors before pregnancy; and/or (III) had newly developed malignant tumors outside the pelvic cavity during pregnancy.

The study was conducted in accordance with the Declaration of Helsinki (as revised in 2013) and was approved by the Ethics Committee of the Beijing ChaoYang Hospital, Capital Medical University, Beijing, China. Informed consents have been attained from patients. 


\section{Data collection}

Patient-related clinical data were collected from the electronic medical record database of the Beijing ChaoYang Hospital, Capital Medical University. Patients' demographic data, obstetric data, and tumor-related data (e.g., age, numbers of pregnancies and deliveries, previous illness history, disease diagnosis, treatment during pregnancy, treatment after delivery, pregnancy outcome, neonatal outcome, maternal outcome, and prognosis of tumor diseases, and so on) were also collected. The relevant data were analyzed to explore the clinical manifestations, pathological characteristics, tumor stage, tumor treatment, and prognosis of newly developed malignant pelvic tumors during pregnancy. The staging of different tumors was based on different criteria. Specifically, gynecological tumor staging was performed according to the FIGO [2009] gynecological tumor staging criteria. Gastric cancer, pancreatic cancer, and non-Hodgkin's lymphoma were classified as stage I, II, III, or IV. Colorectal cancer was classified as stage Duke A, B, C, or D. Tumors of other systems were staged by their tumor criteria.

\section{Management and follow-up methods}

Treatment was scheduled for each patient according to the abnormal conditions found following the prenatal physical examination and tumor screening standards during pregnancy.

Electronic medical record database retrieval, and outpatient and telephone follow-up appointments were conducted. Follow-up visits were scheduled once a month for 6 months after the surgery, once every 2 months for 6 months to 1 year after the surgery, and at a specified followup frequency for related tumors after 1 year. The follow-up period ended in September 2020.

\section{Deferred tumor treatment}

Deferred tumor treatment can be considered for patients $>20$ weeks pregnant with stage $\leq$ IIa cervical cancer, if a family has a a strong wish to proceed with the pregnancy and strongly requests that treatment be deferred. Specific treatment regimens include intravenous chemotherapy for 1 to 2 treatment courses, a cesarean section plus radical hysterectomy after medication withdrawal for over 4 weeks, and drugs to promote fetal lung maturity, which must be administered 1 week before surgery $(10,12,13)$.

\section{Treatment of newly developed malignant pelvic tumors during pregnancy}

The treatment principle for newly developed malignant pelvic tumors during pregnancy is similar to that for nonpregnancy. It is also important to consider the family's and patient's wishes in relation to the pregnancy, and analyze the appropriate deferred treatment for patients with cervical cancer and the continuation of the pregnancy after the surgical treatment of borderline ovarian cancer without compromising the oncological outcomes. Treatment deferral of suspected metastatic tumors should not be considered, and tumor treatment norms should be strictly followed.

\section{Statistical analysis}

Statistical Package for the Social Sciences, version 22.0, software was used for the statistical analysis. The measurement data are expressed as mean \pm standard deviation, and percentages were calculated using enumeration data. The $\chi^{2}$ test was used to compare groups, and the Kaplan-Meier method was used for the survival analysis. Due to the small sample size, a COX risk assessment could not be carried out. A $\mathrm{P}<0.05$ was the $\mathrm{P}$ value for the hypothesis testing.

\section{Results}

\section{Clinical manifestations and pathological category}

A total of 20 patients with newly developed malignant pelvic tumors during pregnancy were enrolled in this study and were allocated to the primary tumor group or metastatic tumor group according to the pathological diagnosis. Of the 14 patients with primary tumors, 3 had malignant tumors and 5 had borderline ovarian tumors. The proportion of borderline ovarian tumors was significantly higher in the pregnant group than the non-pregnant group (65.2\% vs. $9.61 \% ; \mathrm{P}<0.001) .5$ patients had cervical cancer and 1 patient had non-Hodgkin's lymphoma. Of the 6 metastatic ovarian tumor cases, 3 patients had gastric cancer, 2 patients had colon cancer, and 1 patient had pancreatic cancer. $30 \%$ $(6 / 20)$ and $3.53 \%(26 / 737)$ of pregnant and non-pregnant patients had metastatic tumors, respectively, respectively. The proportion of metastatic tumors in pregnant women was significantly higher than the proportion of malignant pelvic tumors in non-pregnant women $\left(\chi^{2}=33.04 ; \mathrm{P}<0.001\right.$, 
Table 1 Pathological and clinical characteristics of newly developed malignant pelvic tumors during pregnancy

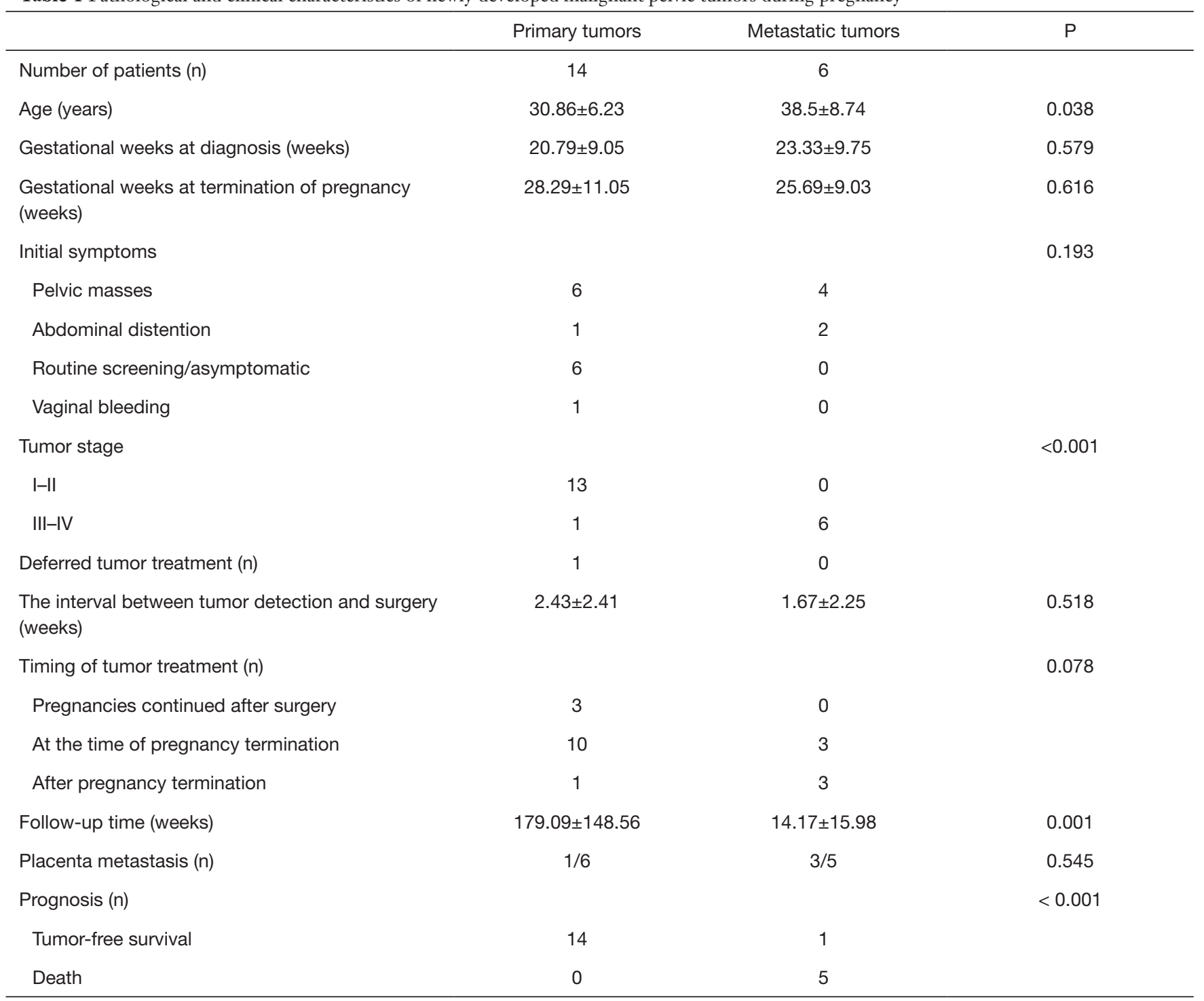

\section{$3.5 \%$ vs. $30 \%)$.}

The clinicopathological characteristics of the patients are set out in Table 1. The age of the patients in the metastatic tumor group was significantly different to that of the primary tumor group $(\mathrm{P}=0.038)$. However, no statistically significant differences were found in the gestation week at diagnosis and the pregnancy-termination week between the 2 groups $(\mathrm{P}=0.59$ and $\mathrm{P}=0.616$, respectively).

In the primary tumor group, in relation to their initial symptoms, $6(42.86 \%$; $6 / 14)$ a pelvic mass, $1(7.14 \%$; $1 / 14)$, abdominal distension, $1(7.14 \% ; 1 / 14)$ vaginal bleeding, and $6(42.86 \% ; 6 / 14)$ patients were asymptomatic but were diagnosed by routine screening. In the metastatic tumor group, $4(42.86 \% ; 6 / 14)$ patients had a pelvic mass, and $2(33.33 \% ; 2 / 6)$, abdominal distension as their initial symptoms. No statistical difference in relation to initial symptoms was found between the 2 groups $(\mathrm{P}=0.193)$.

13 cases in the primary tumor group were stages I-II and 6 cases in the metastatic tumor group were stages IIIIV. A statistical difference was found between the 2 groups $(\mathrm{P}<0.001)$. As Table 2 shows, the histological source of newly developed malignant pelvic tumors during pregnancy was diverse. The placentas of 11 of 20 patients were examined ( 6 cases in the primary tumor group and 5 cases in the metastatic tumor group). One of six cases in the primary tumor group occurred the placental metastasis, and so 
Table 2 Histopathological distribution of 20 cases of newly developed malignant pelvic tumors during pregnancy

\begin{tabular}{|c|c|c|}
\hline Pathology type & Primary tumor group $(\mathrm{n})$ & Metastatic tumor group (n) \\
\hline Gastric signet ring cell carcinoma & 0 & 2 \\
\hline $\begin{array}{l}\text { Poorly differentiated adenocarcinoma of the } \\
\text { colon }\end{array}$ & 0 & 1 \\
\hline Pancreatic cancer & 0 & 1 \\
\hline Cervical squamous cell carcinoma & 2 & 0 \\
\hline Cervical adeno-squamous carcinoma & 1 & 0 \\
\hline Immature teratoma of the ovary & 2 & 0 \\
\hline Mucinous carcinoma of the ovary & 1 & 0 \\
\hline Borderline ovarian tumors & 5 & 0 \\
\hline Ovarian non-Hodgkin's lymphoma & 1 & 0 \\
\hline Total & 14 & 6 \\
\hline
\end{tabular}

$3 / 5$ cases in the metastatic tumor group, respectively. No significant difference was found between the metastatic and primary tumor groups in relation to the placental-metastasis rate of the cancer cells $(\mathrm{P}=0.545)$.

\section{Treatment and prognosis}

The time interval (weeks) from tumor detection to tumor treatment was shorter in the metastatic tumor group than the primary tumor group; however, the difference was not statistically significant $(2.43 \pm 2.41$ vs. $1.67 \pm 2.25 ; \mathrm{P}=0.518)$. In the primary tumor group, 1 patient received deferred treatment, and 3 patients delivery her baby full-time after tumor surgery. Conversely, in the metastatic tumor group, 3 patients underwent tumor therapy at pregnancy termination, 3 patients underwent tumor therapy after pregnancy termination, and no patient received deferred treatment.

Due to the small number of cases in this study, the survival curve and COX risk assessment were not meaningful. The survival analysis showed a significant difference $(\mathrm{P}<0.001)$ in the survival outcomes between the 2 groups. During the follow-up period, all patients in the primary tumor group remained tumor free, but only 1 patient in the metastatic tumor group remained tumor free, and 5 other patients did not survive. Notably, no malignant tumors were found in the 12 newborns during the followup period.

\section{Discussion}

The study collected the clinical data of 20 patients with newly developed malignant pelvic tumors during pregnancy at the Beijing Chao-Yang Hospital, Capital Medical University from the previous 9 years, and systematically compared the clinical characteristics, pathological types, treatment strategies, and clinical prognosis. The results showed that patients in the metastatic tumor group were older than the primary group. Further, the age of patients in the metastatic tumor group was relatively high, the disease was in an advanced stage at the time of diagnosis, the tumor cells had increased placental metastasis, and the prognosis was extremely poor. The common initial symptoms of newly developed malignant pelvic tumors during pregnancy are a pelvic mass and abdominal distension. However, 6 cases in the primary tumor group were found to be asymptomatic 
following routine screening, which suggests that standard prenatal examinations are critical to the detection of malignant pelvic tumors.

Most scholars believe that pregnancy does not increase women's susceptibility to tumors; however, studies have shown that the level of steroid hormones in the body increases during pregnancy, the placenta produces a large amount of heparinase, the level of basal heparin in the body decreases, blood viscosity increases, and cancer cells are prone to mural metastasis. Additionally, the placenta produces a large number of vascular endothelial growth factors that promote tumor growth, and it has been speculated that the risk of pelvic metastasis of malignant tumors in other parts during pregnancy increase $(3,14)$. In this study, a significant difference was found between the percentage of newly developed malignant pelvic tumors during pregnancy and non-pregnancy (30\% vs. $3.53 \%)$. This result is consistent with the inference (14-15). Further, consistent with previous studies, pelvic metastatic tumor cells were found to have poor biological behavior, rapid disease progression, and extremely poor prognosis $(14,15)$. The above results indicate the metastatic malignant tumors of the pelvic cavity threatens the health and life of pregnant women.

Recent studies have shown that common primary gynecological tumors can occur during pregnancy, of which ovarian and cervical cancers are the most common (12). The proportion of borderline ovarian tumors was significantly higher in the pregnant group than the non-pregnant group, but are rarely metastatic and have a good prognosis $(16,17)$. In this study, 5 of 8 cases of primary ovarian tumors were borderline tumors (62.5\% of primary ovarian tumors). This figure was significantly higher than that of the non-pregnant group (for which $9.61 \%$ of ovarian tumors were borderline tumors) for the same period at the hospital. These results are similar to those reported in previous studies $(12,16,17)$.

Current study shows that the pathological types of cervical malignancies are diverse. Research has shown that $85 \%$ of cervical malignant tumors during non-pregnancy are squamous cell carcinoma, followed by adenocarcinoma, adeno-squamous carcinoma, small cell carcinoma, and basal cell carcinoma (10,18-20). Halaska et al. found that nonsquamous cell carcinoma during pregnancy accounted for more higher percentage than non-pregnancy, and differed significantly to the histopathological types of the malignant cervical tumors of the non-pregnant group (20). In the present study, only 2 cases of squamous carcinoma, 1 case of small cell carcinoma, 1 case of adenocarcinoma, 1 case of adeno-squamous carcinoma, thus, non-squamous cell carcinoma accounted for a large proportion (3/5)

The different proportions of histological sources determine treatment options and patient prognosis. According to the previous clinical experience, treatment plans are formulated by the risk of malignance, tissue source of the tumors, and the gestational age. The main prognostic factors were based on clinical stage and histological origin. Metastatic tumors are supposed to be treated by surgical operation as soon as possible, while the therapeutic measures of primary tumor should take the fetus into consideration. Results on the proportion of malignant pelvic tumors during pregnancy are polarized. Additionally, metastatic and borderline tumors account for a much higher proportion than the non-pregnancy, and the prognosis of metastatic tumors is extremely poor. Conversely, borderline ovarian tumors have a good prognosis. Additionally, the histological sources of malignant cervical tumors are diverse, and the proportion of histological types with a poor prognosis is high. Thus, the accurate identification of histological sources is very important for obtaining a good prognosis and is of great significance in reducing maternal mortality.

It is worth noting that tumor markers, which served as an important role of one of the diagnostic criteria of malignant tumor during non-gestational stage, cannot be used to assess the risk of malignance during pregnancy, due to the physiological status of this special phase of life. But still, the value of tumor markers can be monitored dynamically to evaluate the progress of the tumor.

This paper analyzed the clinical characteristics, prognosis, and outcomes of 20 cases of newly developed malignant pelvic tumors during pregnancy at the hospital of this study. The results showed that the histological sources of tumors in pregnant patients are different to those of nonpregnant patients. Additionally, pregnancy may accelerate pelvic metastasis, which develops rapidly during pregnancy, and has an extremely poor prognosis, a high mortality rate, and a certain proportion of placenta metastasis. Thus, clinical decisions should be carefully made for pregnant patients with malignant pelvic tumors to lower the mortality rate and improve the prognosis of patients.

The limits of the study are as follows: (I) the number of the samples in this study is quite small since pelvic malignant tumors during pregnancy are relatively rare; (II) because of the irreconcilable contradiction between ethical issues during pregnancy and diagnostic techniques, an accurate diagnosis and an appropriate treatment is also 
limited. Therefore, a multicentered, large-sampled research is highly recommended to achieve more meaningful and credible conclusions.

\section{Acknowledgments}

Funding: The present study was supported by the Key Projects of Sailing Plan of Beijing Medical Administration (No. ZYLX201713).

\section{Footnote}

Reporting Checklist: The authors have completed the STROBE reporting checklist. Available at https://dx.doi. org/10.21037/tcr-21-717

Data Sharing Statement: Available at https://dx.doi. org/10.21037/tcr-21-717

Conflicts of Interest: All authors have completed the ICMJE uniform disclosure form (available at https://dx.doi. org/10.21037/tcr-21-717). The authors have no conflicts of interest to declare.

Ethical Statement: The authors are accountable for all aspects of the work in ensuring that questions related to the accuracy or integrity of any part of the work are appropriately investigated and resolved. The study was conducted in accordance with the Declaration of Helsinki (as revised in 2013) and was approved by the Ethics Committee of the Beijing Chao-Yang Hospital, Capital Medical University, Beijing, China. Informed consents have been attained from patients.

Open Access Statement: This is an Open Access article distributed in accordance with the Creative Commons Attribution-NonCommercial-NoDerivs 4.0 International License (CC BY-NC-ND 4.0), which permits the noncommercial replication and distribution of the article with the strict proviso that no changes or edits are made and the original work is properly cited (including links to both the formal publication through the relevant DOI and the license). See: https://creativecommons.org/licenses/by-nc-nd/4.0/.

\section{References}

1. Jemal A, Siegel R, Ward E, et al. Cancer statistics, 2007. CA Cancer J Clin 2007;57:43-66.
2. Smith LH, Danielsen B, Allen ME, et al. Cancer associated with obstetric delivery: results of linkage with the California cancer registry. Am J Obstet Gynecol 2003;189:1128-35.

3. Cordeiro CN, Gemignani ML. Gynecologic Malignancies in Pregnancy: Balancing Fetal Risks With Oncologic Safety. Obstet Gynecol Surv 2017;72:184-93.

4. Sakamoto K, Kanda T, Ohashi M, et al. Management of patients with pregnancy-associated gastric cancer in Japan: a mini-review. Int J Clin Oncol 2009;14:392-6.

5. De Carolis S, Grimolizzi F, Garofalo S, et al. Cancer in pregnancy: results of a series of 32 patients. Anticancer Res 2006;26:2413-8.

6. Fruscio R, de Haan J, Van Calsteren K, et al. Ovarian cancer in pregnancy. Best Pract Res Clin Obstet Gynaecol 2017;41:108-17.

7. Albright CM, Wenstrom KD. Malignancies in pregnancy. Best Pract Res Clin Obstet Gynaecol 2016;33:2-18.

8. Voulgaris E, Pentheroudakis G, Pavlidis N. Cancer and pregnancy: a comprehensive review. Surg Oncol 2011;20:e175-85.

9. Han SN, Verheecke M, Vandenbroucke T, et al. Management of gynecological cancers during pregnancy. Curr Oncol Rep 2014;16:415.

10. Perrone AM, Bovicelli A, D'Andrilli G, et al. Cervical cancer in pregnancy: Analysis of the literature and innovative approaches. J Cell Physiol 2019;234:14975-90.

11. Dłuski DF, Mierzyński R,Poniedziaek-Czajkowska E, et al. Ovarian Cancer and Pregnancy-A Current Problem in Perinatal Medicine: A Comprehensive Review. Cancers (Basel) 2020;12:3795.

12. Amant F, Berveiller P, Boere IA, et al. Gynecologic cancers in pregnancy: guidelines based on a third international consensus meeting. Ann Oncol 2019;30:1601-12.

13. Bhatla N, Aoki D, Sharma DN, et al. Cancer of the cervix uteri. Int J Gynaecol Obstet 2018;143 Suppl 2:22-36.

14. Meng J, Kang Y, Cheng H, et al. Misdiagnosed ovarian Krukenberg tumor during pregnancy with virilization. Eur J Gynaecol Oncol 2016;37:587-90.

15. Marbun VMG, Putranto AS. Diagnosis and management of gastric cancer in pregnancy-An evidence-based case report. Int J Surg Case Rep 2020;75:338-44.

16. Frega A, Coluccia AC, Di Martino G, et al. Borderline ovarian tumors, fertility-sparing surgery and pregnancy outcome. Eur Rev Med Pharmacol Sci 2014;18:281-4.

17. Malek-Mellouli M, Ben Amara F, Ferjaoui Mohamed A, et al. Laparoscopic management of borderline malignancy ovarian tumors discovered during pregnancy. Tunis Med 
2013;91:282-3.

18. Fang C, Zhao L, Chen X, et al. The impact of clinicopathologic and surgical factors on relapse and pregnancy in young patients ( $\leq 40$ years old) with borderline ovarian tumors. BMC Cancer 2018;18:1147.

19. Beharee N, Shi Z, Wu D, et al. Diagnosis and treatment of cervical cancer in pregnant women. Cancer Med 2019;8:5425-30.

Cite this article as: Jiang Y, Zhao J, Ding L, Li J, Wang S. Clinical pathological analysis of newly diagnosed malignant pelvic tumors during pregnancy. Transl Cancer Res 2021;10(6):2977-2984. doi: 10.21037/tcr-21-717
20. Halaska MJ, Uzan C, Han SN, et al. Characteristics of patients with cervical cancer during pregnancy: a multicenter matched cohort study. An initiative from the International Network on Cancer, Infertility and Pregnancy. Int J Gynecol Cancer 2019. [Epub ahead of print]. doi: 10.1136/ijgc-2018-000103.

(English Language Editor: L. Huleatt) 